\title{
The association of alcohol and smoking with CKD in a Japanese nationwide cross-sectional survey
}

\author{
Ayako Matsumoto ${ }^{1}$, Yasuyuki Nagasawa ${ }^{1}$, Ryohei Yamamoto $^{2,3}$, Maki Shinzawa ${ }^{3}$, Yukiko Hasuike ${ }^{1}$, \\ Takahiro Kuragano ${ }^{1}$, Yoshitaka Isaka ${ }^{3}$, Takeshi Nakanishi ${ }^{1}$, Kunitoshi Iseki ${ }^{4}$, Kunihiro Yamagata ${ }^{4}$, \\ Kazuhiko Tsuruya $^{4}$, Hideaki Yoshida ${ }^{4}$, Shouichi Fujimoto ${ }^{4}$, Koichi Asahi ${ }^{4}$, Toshiki Moriyama ${ }^{2,3,4}$ \\ and Tsuyoshi Watanabe ${ }^{4}$
}

Chronic kidney disease (CKD) is characterized by a reduced glomerular filtration rate (GFR) and proteinuria. Modifiable lifestyle factors such as smoking and alcohol contribute to CKD. Recent cohort studies have shown that moderate alcohol consumption attenuates the decline of the GFR and smoking has been previously shown to be associated with CKD. However, the association of smoking and alcohol consumption on CKD is not entirely clear. To examine whether there is evidence to assume that smoking is an effective modifier of the association between CKD and alcohol consumption, we conducted a cross-sectional study of a population of people who presented for a health checkup under a program that targets the insured population aged $\geqq 40$ years using data from the Specific Health Check and Guidance in Japan between April 2008 and March 2009. Of the 506807 participants aged $\geqslant 40$ years, $292013(57.6 \%)$ were included in the present analysis. Outcomes were kidney dysfunction, as an eGFR of $<60 \mathrm{ml} / \mathrm{min} / 1.73 \mathrm{~m}^{2}$, and proteinuria. In nonsmokers, drinking a small amount was associated with a lower prevalence of proteinuria, but in smokers, the association between alcohol and proteinuria was not observed. The analysis regarding eGFR $<60 \mathrm{ml} / \mathrm{min} / 1.73 \mathrm{~m}^{2}$ revealed that in both smokers and nonsmokers, alcohol consumption was inversely associated with the risk of CKD. Mild to moderate alcohol consumption might be associated with a lower risk of CKD (proteinuria and eGFR), especially among nonsmokers, because smoking might have modified the potential benefits of alcohol to prevent CKD.

Hypertension Research (2017) 40, 771-778; doi:10.1038/hr.2017.25; published online 9 March 2017

Keywords: alcohol consumption; CKD; proteinuria; smoking

\section{INTRODUCTION}

The concept of chronic kidney disease (CKD) was developed because $\mathrm{CKD}$ has a strong relationship with cardiovascular diseases (CVDs) as well as end-stage renal diseases (ESRDs). ${ }^{1-3}$ Originally, CKD was defined by proteinuria and glomerular filtration rates (GFRs). ${ }^{4}$

Similar to CVD, modifiable lifestyle factors, such as smoking, 5 alcohol, ${ }^{7}$ sleep ${ }^{8}$ and exercise, ${ }^{9}$ contribute to CKD. ${ }^{10}$

Smoking has been previously shown to be associated with renal disease. ${ }^{11-14}$ A number of biological mechanisms by which smoking can result in kidney damage have been identified, including the promotion of renal atherosclerosis, ${ }^{15}$ alterations in systemic and renal hemodynamics ${ }^{16}$ and effects on endothelial function. ${ }^{17}$

Alcohol consumption is one of the main modifiable lifestyle factors associated with CVD and its risk factors, including hypertension, ${ }^{18}$ diabetes, ${ }^{19}$ ischemic heart disease ${ }^{20}$ and stroke. ${ }^{21}$ Heavy drinkers are at a higher risk of these diseases, whereas mild to moderate alcohol consumption, generally corresponding to 1-2 drinks (15-30 g of alcohol) per day or less, is associated with a lower incidence of these diseases and a lower all-cause mortality. ${ }^{22}$ In 'Healthy Japan 21', the Japanese Ministry of Health and Welfare reported that consuming a mean amount of $\sim 20 \mathrm{~g}$ of absolute alcohol per day represented 'moderate drinking. ${ }^{23}$ For renal disease, it remains controversial whether heavy alcohol consumption affects renal prognosis. ${ }^{24-26}$ Recent cohort studies have shown that moderate alcohol consumption attenuates the decline of GFR. ${ }^{27}$

Because various background factors can affect the relevance of alcohol consumption and $\mathrm{CKD}$, a variety of research reports have been reported. For example, joint exposure to both current smoking and heavy drinking has been associated with higher odds of CKD than their individual effects. ${ }^{28}$ However, the association of smoking and alcohol consumption on CKD is not entirely clear.

The aim of the present cross-sectional survey of 292013 individuals undergoing the Specific Health Check and Guidance in Japan was to examine whether there is evidence to assume that smoking modifies the potential benefits of alcohol to prevent CKD.

\footnotetext{
${ }^{1}$ Department of Internal Medicine, Division of Kidney and Dialysis, Hyogo College of Medicine, Hyogo, Japan; ${ }^{2}$ Osaka University Health Care Center, Osaka, Japan; ${ }^{3}$ Department of Geriatric Medicine and Nephrology, Osaka University Graduate School of Medicine, Osaka, Japan and ${ }^{4}$ Steering Committee for the 'Research on the Positioning of Chronic Kidney Disease in Specific Health Check and Guidance in Japan', Tokyo, Japan

Correspondence: Dr T Moriyama, Osaka University Healthcare Center, 1-17 Machikaneyama-cho, Toyonaka 560-0043, Japan.

E-mail: moriyama@wellness.hss.osaka-u.ac.jp

Received 5 December 2016; revised 11 January 2017; accepted 30 January 2017; published online 9 March 2017
} 


\section{METHODS}

\section{Study population}

The present nationwide cross-sectional survey included members of the general Japanese population who underwent the Specific Health Check and Guidance in Japan between April 2008 and March 2009. As a recent survey indicated that the prevalence of diabetes, hypertension, dyslipidemia, obesity and metabolic syndrome had been increasing in Japan, the Ministry of Health, Labor and Welfare of Japan developed a systematic health-care strategy that involves specific health checkups and targets members of the insured population who are aged $\geqslant 40$ years. This strategy aims to detect and manage CKD at an earlier stage. We analyzed the data of individuals who were receiving the Health Check and Guidance in 8 prefectures (Miyagi, Fukushima, Ibaraki, Tokyo, Niigata, Osaka, Fukuoka and Okinawa). Of the 506807 participants aged $\geqslant 40$ years (Miyagi 16 640, Fukushima 50 304, Ibaraki 39 775, Tokyo 40 278, Niigata 58 882, Osaka 25 615, Fukuoka 149785 and Okinawa 125 528), 292013 (57.6\%) were included in the present analysis; 214794 (42.4\%) participants with missing data were excluded (among them 65577 participants who were excluded because of a lack of responses to questions related to smoking or drinking). The study protocol was approved by the ethics committee at Fukushima Medical University (No. 715) and Osaka University Hospital (No. 13085). We deleted the patient's name, date of birth (except the month and year of birth) and patient ID in each facility. Then, we labeled the patients in each facility and made a correspondence table, and the table was kept in the locked area.

\section{Measurements}

Demographic, physical and laboratory data included age, sex, body mass index (weight $(\mathrm{kg}) /$ height $^{2}\left(\mathrm{~m}^{2}\right)$ ), systolic blood pressure, diastolic blood pressure, mean arterial pressure (diastolic blood pressure+(systolic blood pressure - diastolic blood pressure)/3), pulse pressure (systolic blood pressure - diastolic blood pressure), serum concentration of creatinine (enzymatic method), and levels of hemoglobin Alc, fasting blood sugar, triglycerides, high-density lipoprotein cholesterol (HDL-C), low-density lipoprotein cholesterol, uric acid and urinary protein, as detected by a dipstick test. Proteinuria was defined as $\geqslant 1$ urinary proteins. The estimated glomerular filtration rate (eGFR) was calculated using the following equation: eGFR $\left(\mathrm{ml} / \mathrm{min} / 1.73 \mathrm{~m}^{2}\right)=194 \times$ age $\quad$ (years $)^{-0.287} \times$ serum creatinine $(\mathrm{mg} / \mathrm{dl})^{-1.094}(\times 0.739$, if female $){ }^{29}$ Hypertension is defined as follows, systolic blood pressure $\geqq 140 \mathrm{mmHg}$ or diastolic blood pressure $\geqq 90 \mathrm{mmHg}$. ${ }^{30}$ Diabetes is defined as follows, fasting plasma glucose level $\geqslant 126 \mathrm{mg} / \mathrm{dl}$ and hemoglobin Alc $\geqslant 6.1 \% .{ }^{31}$ Dyslipidemia is defined as follows, low-density lipoprotein cholesterol $\geqq 140 \mathrm{mg} / \mathrm{dl}$ or HDL-C $<40 \mathrm{mg} / \mathrm{dl}$ or triglycerides $\geqq 150 \mathrm{mg} / \mathrm{dl}^{32}$

Information regarding lifestyle, use of current medications for comorbidities and past medical history was based on self-reported standard questionnaires that all participants were required to complete at the time of their check-up. Alcohol consumption was ascertained by asking the following two questions: 'How often do you drink alcoholic beverages? (1) Every day, (2) occasionally or (3) rarely' and 'How many alcoholic beverages do you drink on the days that you do drink? (Approximately $500 \mathrm{ml}$ beer, $80 \mathrm{ml}$ 'shochu' (a Japanese liquor similar to vodka), $60 \mathrm{ml}$ whiskey or $240 \mathrm{ml}$ wine were assumed to constitute 1 standard drink.) (1) $\leqq 1$ Drink per day, (2) $1-2$ drinks per day, (3) 2-3 drinks per day or (4) $\geqq 3$ drinks per day'. Each drink was calculated using $20 \mathrm{~g}$ of ethanol consumption. We categorized alcohol consumption as follows: drink score (0) rare, (1) occasional, (2) ethanol intake $\leqq 19 \mathrm{~g}$ per day, (3) ethanol intake $20-39 \mathrm{~g}$ per day, (4) ethanol intake $40-59 \mathrm{~g}$ per day and (5) ethanol intake $\geqq 60 \mathrm{~g}$ per day. Smoking status was evaluated by positive answers to the question 'Do you smoke now?' A past history of cardiac disease was defined by a positive answer to the question 'Have you been diagnosed with heart disease?' Similarly, past history of stroke was also defined by a positive answer to the question 'Have you been diagnosed with stroke?' and a past history of kidney disease was defined by a positive answer to the question 'Have you been diagnosed with kidney disease?' Current drug history was defined by a positive answer to the question 'Do you take antihypertensive medications now?', 'Do you take insulin injection or antihyperglycemia medications now?' and 'Do you take anticholesteremic medications now?'
All data were collected and managed at an independent non-profit data center in the Japan Clinical Research Support Unit.

\section{Statistical analysis}

Continuous variables were expressed as the mean \pm s.d. or median (interquartile range) as appropriate, and categorical variables were expressed as a number (proportion). Serum triglyceride levels were logarithmically transformed because of their skewed distribution. The clinical characteristics of subjects with different drink scores were compared using analysis of variance, the Kruskal-Wallis test or $\chi^{2}$ test, as appropriate. To assess whether smoking is an effect modifier of the association between drink score and proteinuria, the odds ratio of each drink score was calculated using univariate and multivariate logistic regression models, dividing it into smoking and nonsmoking, with the presence of proteinuria as the dependent variable. To assess whether smoking is an effect modifier of the association between drink scores and eGFRs, the odds ratio of each drink score was calculated using univariate and multivariate logistic regression models, dividing it into smoking and nonsmoking, with the presence of eGFR $<60\left(\mathrm{ml} / \mathrm{min} / 1.73 \mathrm{~m}^{2}\right)$ as the dependent variable. Multivariate models included age, body mass index, systolic blood pressure, diastolic blood pressure, smoking status, drinking status, past history of heart disease, past history of stroke, levels of hemoglobin Alc, serum triglyceride concentration, HDL cholesterol and uric acid as covariates, in addition to the drink score.

We defined kidney dysfunction, one of the outcomes, as an eGFR $<60 \mathrm{ml} / \mathrm{min} / 1.73 \mathrm{~m}^{2}$ because recent reports have demonstrated that an eGFR $<60 \mathrm{ml} / \mathrm{min} / 1.73 \mathrm{~m}^{2}$ is a risk factor for CVD and end-stage renal disease (ESRD), resulting in the establishment of CKD stage 3a. We assessed proteinuria (defined as $\geqq 1+$ urinary protein by dipstick test) as the other outcome.

All $P$-values were based on two-sided tests of significance, and $P<0.05$ was considered statistically significant. Statistical analyses were performed using Stata version 11.0 (Stata, College Station, TX, USA) and R version 3.0.2 (The R Foundation for Statistical Computing, http://www.r-project.org/).

\section{RESULTS}

The clinical characteristics of the 117692 men and 174321 women stratified according to smokers and nonsmokers are shown in Tables 1 and 2, respectively. In men, smokers tended to drink more alcohol than nonsmokers, and smokers were younger, had lower body mass index, lower systolic and diastolic blood pressure, lower serum HDL-C and low-density lipoprotein cholesterol level, higher eGFR, higher positive proteinuria, higher triglyceride levels and higher hemoglobin A1c. In male nonsmokers, past histories of heart disease, stroke and kidney disease, current drug history of antihypertensive and anticholesteremic medications and prevalence of hypertension were higher. In the women, almost all were nonsmokers (94.1\%), and similar trends as in the men were observed. In addition, uric acid and past history of kidney disease were slightly higher in female smokers, and prevalence of dyslipidemia was higher in female nonsmokers.

We examined the relationship between alcohol consumption and proteinuria stratified by smoking. In both genders, univariate and multivariate model analyses demonstrated that alcohol consumption was associated with proteinuria (Tables 3 and 4). The multivariate analysis of proteinuria revealed that in nonsmokers, a small amount of drinking was associated with a lower prevalence of proteinuria, but in smokers, the association of alcohol consumption and proteinuria was not observed. In male nonsmokers, a drink score of 2 and 3 had a significant association with a lower prevalence of proteinuria (vs. a drink score of 0 , multivariate-adjusted odds ratio (OR) 0.90 (95\% confidence interval (CI) $0.83-0.98), P=0.012$ for drink score 2, multivariate-adjusted OR 0.87 (95\% CI 0.79-0.94), $P=0.001$ for drink score 3 ), although in smokers, alcohol was not associated with a lower prevalence of proteinuria. In female nonsmokers, a drink score of 1 had a significant association with a lower prevalence 
Table 1 The clinical characteristics of 117692 men stratified according to smokers and nonsmorkers

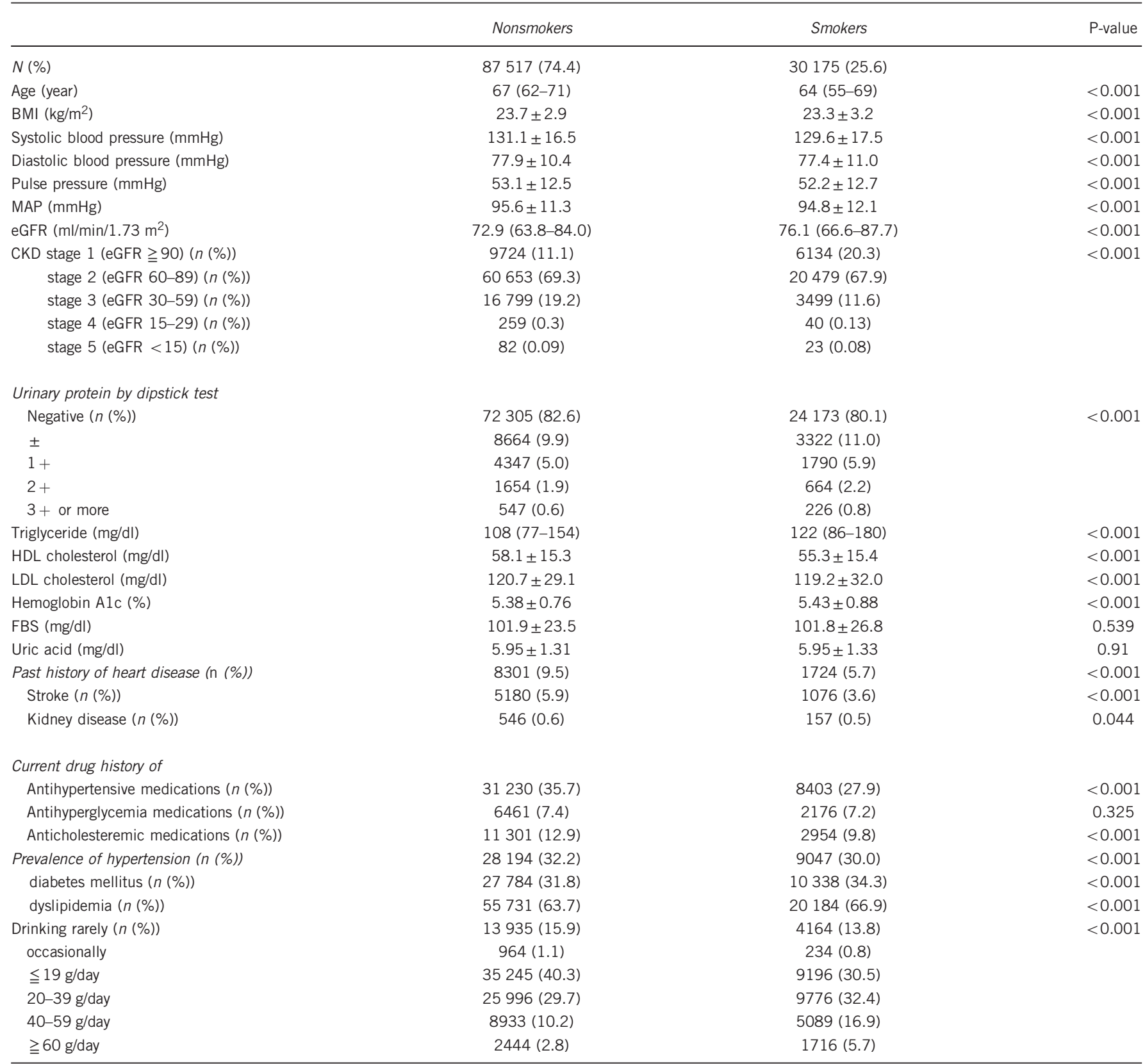

Abbreviations: BMI, body mass index; CKD, chronic kidney disease; eGFR, estimated glomerular filtration rate; FBS, fasting blood sugar; HDL, high-density lipoprotein; LDL, low-density lipoprotein; MAP, mean arterial pressure.

Mean \pm s.d., median (25-75\%).

of proteinuria ( $v s$. a drink score of 0 , multivariate-adjusted OR 0.75 (95\% CI 0.59-0.96), $P=0.021$ ), although in smokers, the association of alcohol and proteinuria was not observed.

We examined the relationship between alcohol consumption and eGFR $<60 \mathrm{ml} / \mathrm{min} / 1.73 \mathrm{~m}^{2}$ stratified by smoking. In both genders, univariate and multivariate model analyses demonstrated that alcohol consumption was associated with a lower prevalence of eGFR $<60 \mathrm{ml} / \mathrm{min} / 1.73 \mathrm{~m}^{2}$ (Tables 5 and 6). The multivariate analysis of eGFR $<60 \mathrm{ml} / \mathrm{min} / 1.73 \mathrm{~m}^{2}$ revealed that in both smokers and nonsmokers, alcohol consumption was inversely associated with the risk of chronic kidney disease. In male nonsmokers, drink scores from 1 to 5 had a significant association with a lower prevalence of
eGFR $<60 \mathrm{ml} / \mathrm{min} 1.73 \mathrm{~m}^{2}$ (vs. a drink score of 0 , multivariateadjusted OR 0.71 (95\% CI 0.59-0.85), $P<0.001$ for drink score 1 , 0.90 (0.85-0.95), $P<0.001$ for drink score 2, $0.62(0.58-0.65)$, $P<0.001$ for drink score 3, $0.54(0.49-0.58), P<0.001$ for drink score 4 and $0.57(0.49-0.65), P<0.001$ for drink score 5$)$, and in smokers, drink scores from 1 to 5 had a significant association with a lower prevalence of eGFR $<60 \mathrm{ml} / \mathrm{min} / 1.73 \mathrm{~m}^{2}$ ( $v s$. a drink score of 0 , multivariate-adjusted OR 0.58 (95\% CI 0.36-0.96), $P=0.033$ for drink score 1, 0.80 (0.71-0.90), $P<0.001$ for drink score 2, $0.61(0.54-0.69)$, $P<0.001$ for drink score 3, 0.45 (0.39-0.53), $P<0.001$ for drink score 4 and $0.39(0.31-0.50), P<0.001$ for drink score 5). In female nonsmokers, drink scores from 1 to 5 had a significant association 
Table 2 The clinical characteristics of 174321 women stratified according to smokers and nonsmorkers

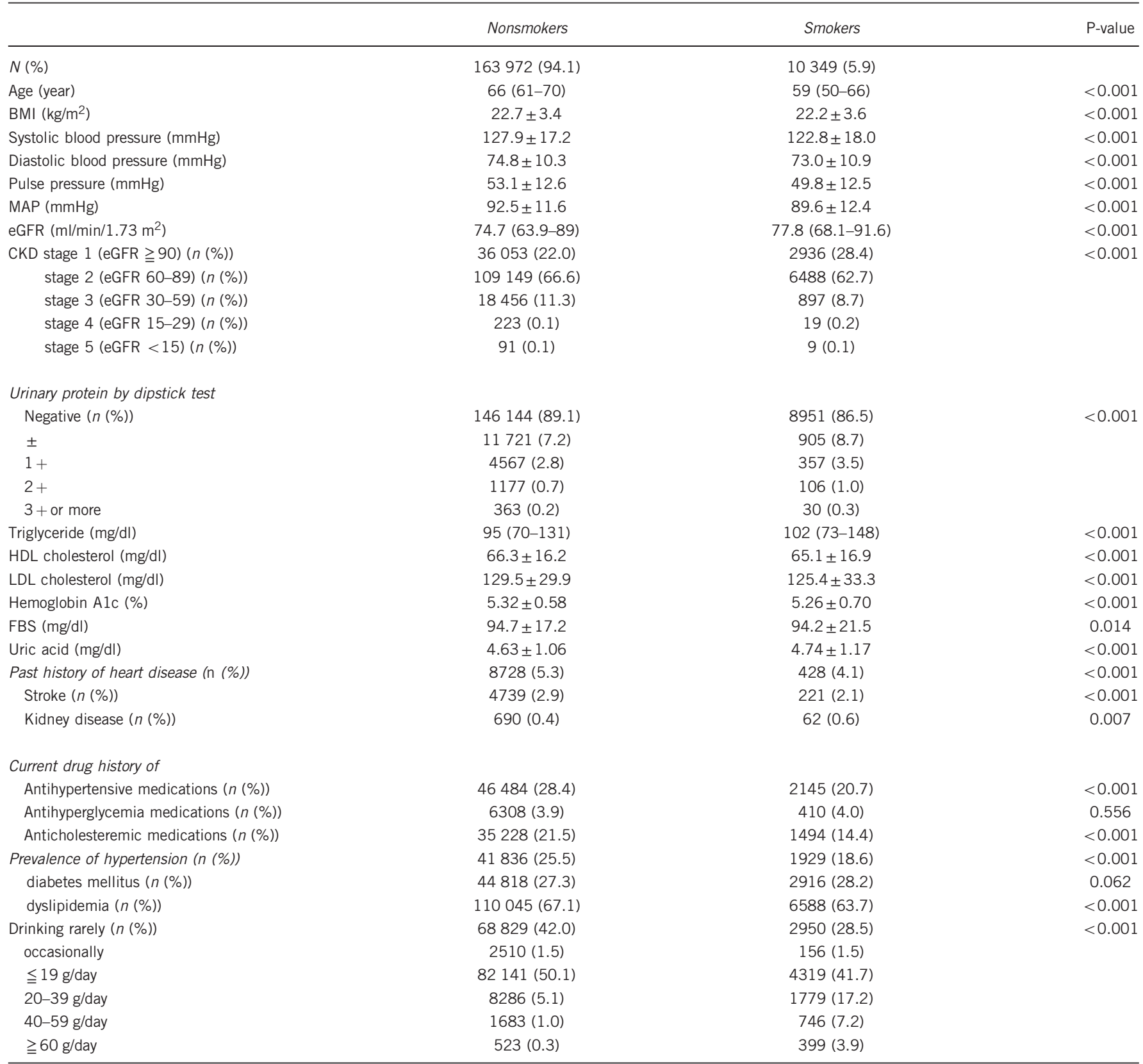

Abbreviations: BMI, body mass index; CKD, chronic kidney disease; eGFR, estimated glomerular filtration rate; FBS, fasting blood sugar; HDL, high-density lipoprotein; LDL, low-density lipoprotein; MAP, mean arterial pressure.

Mean \pm s.d., median (25-75\%).

with a lower prevalence of eGFR $<60 \mathrm{mlml} / \mathrm{min} / 1.73 \mathrm{~m}^{2}$ (vs. a drink score of 0 , multivariate-adjusted OR 0.74 (95\% CI $0.64-0.86$ ), $P<0.001$ for drink score $1,0.94(0.91-0.97), P=0.001$ for drink score 2, 0.66 (0.60-0.72), $P<0.001$ for drink score $3,0.53(0.43-0.65)$, $P<0.001$ for drink score 4 and 0.35 (0.23-0.54), $P<0.001$ for drink score 5 ), and in smokers, drink scores from 3 to 5 had a significant association with a lower prevalence of eGFR $<60 \mathrm{ml} / \mathrm{min} / 1.73 \mathrm{~m}^{2}$ (vs. a drink score of 0 , multivariate-adjusted OR 0.55 (95\% CI 0.42-0.72), $P<0.001$ for drink score 3, $0.51(0.34-0.75), P=0.001$ for drink score 4 , and $0.27(0.14-0.54), P<0.001$ for drink score 5). In female smokers, the association between drink scores of 1 and 2 and a lower prevalence of eGFR $<60 \mathrm{ml} / \mathrm{min} / 1.73 \mathrm{~m}^{2}$ was not observed.
We also calculated the odds ratio of each drink score using univariate and multivariate logistic regression models, excluding the person with the past medical history (heart disease, stroke and kidney disease), dividing it into smoking and nonsmoking, with the presence of proteinuria as the dependent variable (Supplementary Tables 1 and 2). Furthermore, we calculated the odds ratio of each drink score using univariate and multivariate logistic regression models, excluding the person with the past medical history (heart disease, stroke and kidney disease), dividing it into smoking and nonsmoking, with the presence of eGFR $<60\left(\mathrm{mlml} / \mathrm{min} / 1.73 \mathrm{~m}^{2}\right)$ as the dependent variable (Supplementary Tables 3 and 4). Compared with Tables 3,4,5,6, these results were almost similar. 
Table 3 The associations of alcohol consumption and proteinuria in men stratified by smoking

\begin{tabular}{|c|c|c|c|c|c|c|c|c|}
\hline & \multicolumn{4}{|c|}{ Nonsmokers } & \multicolumn{4}{|c|}{ Smokers } \\
\hline & \multicolumn{2}{|c|}{ Univariate model } & \multicolumn{2}{|c|}{ Multivariate mode ${ }^{\circledR}$} & \multicolumn{2}{|c|}{ Univariate model } & \multicolumn{2}{|c|}{ Multivariate mode ${ }^{A}$} \\
\hline & OR $(95 \% \mathrm{Cl})$ & P-value & OR $(95 \% \mathrm{Cl})$ & P-value & OR $(95 \% \mathrm{Cl})$ & P-value & OR $(95 \% \mathrm{Cl})$ & P-value \\
\hline Drinking rarely $(n(\%))$ & Reference & & Reference & & Reference & & Reference & \\
\hline occasionally & $0.87(0.68-1.12)$ & 0.228 & $0.82(0.62-1.08)$ & 0.164 & $0.68(0.40-1.17)$ & 0.163 & $0.78(0.43-1.39)$ & 0.398 \\
\hline $40-59 \mathrm{~g} / \mathrm{day}$ & $0.95(0.86-1.05)$ & 0.299 & $0.96(0.86-1.07)$ & 0.467 & $0.99(0.86-1.14)$ & 0.917 & $0.98(0.83-1.16)$ & 0.844 \\
\hline$\geqq 60$ g/day & $1.05(0.90-1.23)$ & 0.506 & $1.09(0.92-1.28)$ & 0.33 & $1.11(0.92-1.34)$ & 0.271 & $1.06(0.85-1.32)$ & 0.595 \\
\hline Age $(\times 10$ years $)$ & $1.22(1.18-1.26)$ & $<0.001$ & $1.17(1.12-1.22)$ & $<0.001$ & $1.19(1.14-1.25)$ & $<0.001$ & $1.14(1.08-1.20)$ & $<0.001$ \\
\hline BMI $\left(\times 10 \mathrm{~kg} / \mathrm{m}^{2}\right)$ & $2.98(2.75-3.23)$ & $<0.001$ & $2.07(1.88-2.28)$ & $<0.001$ & $2.11(1.88-2.38)$ & $<0.001$ & $1.64(1.42-1.90)$ & $<0.001$ \\
\hline Systolic BP (mmHg) & $1.02(1.02-1.02)$ & $<0.001$ & $1.02(1.01-1.02)$ & $<0.001$ & $1.02(1.01-1.02)$ & $<0.001$ & $1.02(1.01-1.02)$ & $<0.001$ \\
\hline Uric acid (mg/dl) & $1.16(1.14-1.18)$ & $<0.001$ & $1.15(1.12-1.17)$ & $<0.001$ & $1.12(1.09-1.16)$ & $<0.001$ & $1.15(1.11-1.19)$ & $<0.001$ \\
\hline \multicolumn{9}{|l|}{ Past history of } \\
\hline Heart disease & $1.57(1.46-1.69)$ & $<0.001$ & $1.40(1.28-1.52)$ & $<0.001$ & $1.80(1.56-2.07)$ & $<0.001$ & $1.63(1.39-1.90)$ & $<0.001$ \\
\hline Stroke & $1.85(1.70-2.02)$ & $<0.001$ & $1.57(1.43-1.73)$ & $<0.001$ & $1.81(1.52-2.16)$ & $<0.001$ & $1.46(1.20-1.77)$ & $<0.001$ \\
\hline
\end{tabular}

Abbreviations: $\mathrm{BMI}$, body mass index; $\mathrm{BP}$, blood pressure; $\mathrm{Cl}$, confidence interval; HDL-C, high-density lipoprotein cholesterol; OR, odds ratio.

aAdjusted for alcohol consumption, age, body mass index, mean arterial pressure, triglyceride level, HDL cholesterol level, hemoglobin Alc, uric acid, past history of heart disease and stroke.

Table 4 The associations of alcohol consumption and proteinuria in women stratified by smoking

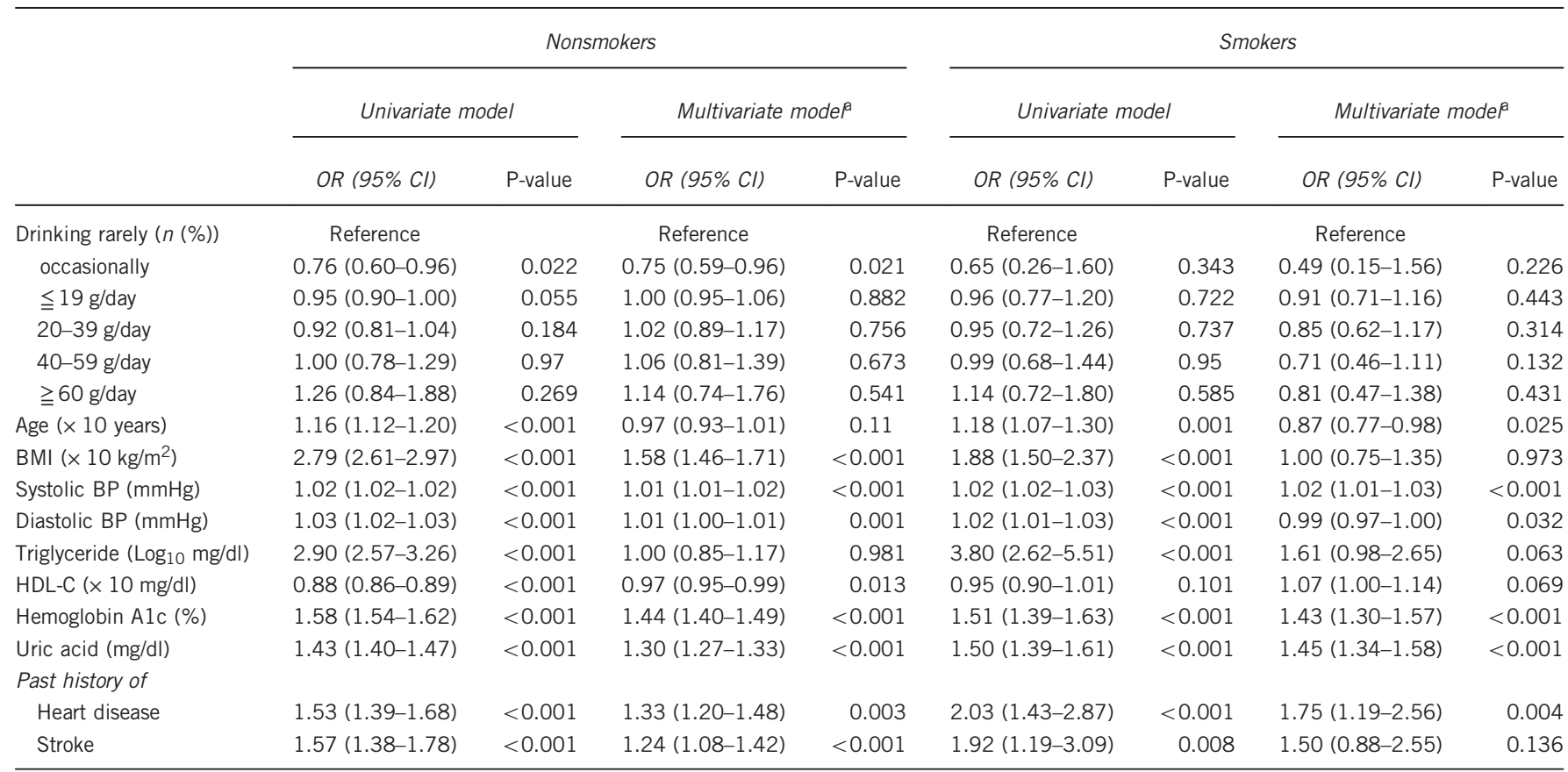

Abbreviations: BMI, body mass index; BP, blood pressure; Cl, confidence interval; HDL-C, high-density lipoprotein cholesterol; OR, odds ratio.

${ }^{a}$ Adjusted for alcohol consumption, age, body mass index, mean arterial pressure, triglyceride level, HDL cholesterol level, hemoglobin A1c, uric acid, past history of heart disease and stroke.

\section{DISCUSSION}

In the present cross-sectional study of 292013 individuals undergoing the Specific Health Check and Guidance in Japan, it was observed that in nonsmokers, a small amount of drinking was associated with a lower prevalence of proteinuria. However, in smokers, the association between alcohol and proteinuria was not observed. In the analysis 
Table 5 The associations of alcohol consumption and eGFR $<60 \mathrm{ml} / \mathrm{min} / 1.73 \mathrm{~m}^{2}$ in men stratified by smoking

\begin{tabular}{|c|c|c|c|c|c|c|c|c|}
\hline & \multicolumn{4}{|c|}{ Nonsmokers } & \multicolumn{4}{|c|}{ Smokers } \\
\hline & \multicolumn{2}{|c|}{ Univariate model } & \multicolumn{2}{|c|}{ Multivariate mode ${ }^{\beta}$} & \multicolumn{2}{|c|}{ Univariate model } & \multicolumn{2}{|c|}{ Multivariate mode ${ }^{\beta}$} \\
\hline & OR $(95 \% \mathrm{Cl})$ & P-value & OR $(95 \% \mathrm{Cl})$ & P-value & OR $(95 \% \mathrm{Cl})$ & P-value & OR $(95 \% \mathrm{Cl})$ & P-value \\
\hline Drinking rarely $(n(\%))$ & Reference & & Reference & & Reference & & Reference & \\
\hline occasionally & $0.81(0.69-0.96)$ & 0.012 & $0.71(0.59-0.85)$ & $<0.001$ & $0.63(0.41-0.98)$ & 0.038 & $0.58(0.36-0.96)$ & 0.033 \\
\hline 20-39 g/day & $0.70(0.66-0.73)$ & $<0.001$ & $0.62(0.58-0.65)$ & $<0.001$ & $0.76(0.68-0.84)$ & $<0.001$ & $0.61(0.54-0.69)$ & $<0.001$ \\
\hline 40-59 g/day & $0.56(0.52-0.60)$ & $<0.001$ & $0.54(0.49-0.58)$ & $<0.001$ & $0.51(0.45-0.59)$ & $<0.001$ & $0.45(0.39-0.53)$ & $<0.001$ \\
\hline$\geqq 60$ g/day & $0.51(0.45-0.58)$ & $<0.001$ & $0.57(0.49-0.65)$ & $<0.001$ & $0.38(0.30-0.47)$ & $<0.001$ & $0.39(0.31-0.50)$ & $<0.001$ \\
\hline Age (× 10 years $)$ & $2.06(2.01-2.12)$ & $<0.001$ & 2.26 (2.19-2.34) & $<0.001$ & $2.51(2.38-2.64)$ & $<0.001$ & 2.76 (2.59-2.94) & $<0.001$ \\
\hline BMI $\left(\times 10 \mathrm{~kg} / \mathrm{m}^{2}\right)$ & $1.72(1.63-1.82)$ & $<0.001$ & $1.32(1.23-1.41)$ & $<0.001$ & $1.64(1.47-1.82)$ & $<0.001$ & $1.55(1.35-1.78)$ & $<0.001$ \\
\hline Systolic BP (mmHg) & $1.01(1.00-1.01)$ & $<0.001$ & $1.00(0.99-1.00)$ & $<0.001$ & $1.01(1.00-1.01)$ & $<0.001$ & $1.00(0.99-1.00)$ & 0.247 \\
\hline Uric acid (mg/dl) & $1.56(1.54-1.59)$ & $<0.001$ & $1.66(1.63-1.68)$ & $<0.001$ & $1.50(1.46-1.54)$ & $<0.001$ & $1.62(1.57-1.68)$ & $<0.001$ \\
\hline \multicolumn{9}{|l|}{ Past history of } \\
\hline Heart disease & $1.71(1.63-1.80)$ & $<0.001$ & $1.39(1.31-1.47)$ & $<0.001$ & $2.28(2.02-2.57)$ & $<0.001$ & $1.64(1.42-1.88)$ & $<0.001$ \\
\hline Stroke & $1.74(1.63-1.85)$ & $<0.001$ & $1.35(1.26-1.45)$ & $<0.001$ & $2.15(1.85-2.50)$ & $<0.001$ & 1.39 (1.17-1.65) & $<0.001$ \\
\hline
\end{tabular}

Abbreviations: $\mathrm{BMI}$, body mass index; $\mathrm{BP}$, blood pressure; $\mathrm{Cl}$, confidence interval; eGFR, estimated glomerular filtration rate; HDL-C, high-density lipoprotein cholesterol; OR, odds ratio.

${ }^{a}$ Adjusted for alcohol consumption, age, body mass index, mean arterial pressure, triglyceride level, HDL cholesterol level, hemoglobin A1c, uric acid, past history of heart disease and stroke.

regarding eGFR $<60 \mathrm{ml} / \mathrm{min} / 1.73 \mathrm{~m}^{2}$, it was observed that in both smokers and nonsmokers, alcohol consumption was inversely associated with the risk of $\mathrm{CKD}$; however, in female smokers, the association of drink scores 1 and 2 with a lower prevalence of eGFR $<60 \mathrm{ml} / \mathrm{min} / 1.73 \mathrm{~m}^{2}$ was not observed.

Although alcohol consumption has been found to be protective for $\mathrm{CVD},{ }^{20}$ the relationship between alcohol consumption and kidney disease is complex and controversial. On the one hand, alcohol consumption has been associated with the risk of CKD. ${ }^{28,33}$ A population-based cohort study in the United States reported that drinking $\geqslant 4$ servings daily was independently associated with an increased CKD risk ${ }^{28}$ (each serving of beer, wine and liquor was considered to contain 12.96, 11.48 and $14.00 \mathrm{~g}$ of ethanol, respectively ${ }^{34}$ ). A case-control study in the United States reported that the consumption of more than two alcoholic drinks per day was associated with an increased risk of ESRD and that a lower intake of alcohol did not appear to be harmful. ${ }^{33}$

On the other hand, alcohol consumption has been inversely associated with the risk of CKD. ${ }^{25-27,35-37}$ A prospective cohort study of 11023 initially healthy male physicians in the United States reported a significantly lower risk of renal dysfunction (defined as serum creatinine $\geqslant 1.5 \mathrm{mg} / \mathrm{dl}$ and eGFR $\leqslant 55 \mathrm{ml} / \mathrm{min}$ ) in those who consumed at least 7 drinks weekly. ${ }^{37} \mathrm{~A}$ prospective cohort study in China reported a significant inverse relationship between alcohol consumption and risk of ESRD in men and a stronger relationship in those who had more than 21 drinks weekly. ${ }^{25}$ A prospective cohort study in Australia reported a significantly reduced risk of CKD (defined as eGFR $<60 \mathrm{ml} / \mathrm{min} / 1.73 \mathrm{~m}^{2}$ ) in men who consumed at least $30 \mathrm{~g}$ of alcohol daily. ${ }^{26}$ A prospective cohort study in Japan reported that in men aged $\geqq 40$ years, an average daily alcohol consumption of $<20 \mathrm{~g}$ /day decreased the risk of CKD compared with nondrinkers, whereas an average daily alcohol consumption of $\geqq 20 \mathrm{~g} /$ day was not associated with the risk of CKD. ${ }^{27}$
In our results, mild to moderate alcohol consumption was associated with a lower incidence of proteinuria, and this finding was consistent with previous publications. However, in smokers, the association of alcohol with proteinuria was not observed, and hence this association might be no longer significant.

Furthermore, alcohol consumption was inversely associated with the risk of decreased eGFR, but in female smokers, the association between mild to moderate alcohol consumption and a lower prevalence of eGFR $<60 \mathrm{ml} / \mathrm{min} / 1.73 \mathrm{~m}^{2}$ was not observed. We suspect that smoking may modify the potential benefits of alcohol to prevent CKD. Alcohol also has an adverse effect on driving safety and can increase violent behavior, liver dysfunction and some cancers; therefore, the results regarding alcohol intake should be interpreted carefully. Nevertheless, in nonsmokers, mild to moderate alcohol consumption may not need to be prohibited, at least regarding its effects on kidney function. On the other hand, alcohol intake may not be recommended for smokers.

Some clinical studies have indicated the mechanisms involved with alcohol consumption, for example, that moderate consumption may be associated with increased HDL and plasma concentration of endogenous tissue-type plasminogen activator, ${ }^{38,39}$ thereby protecting against atherosclerosis. However, the mechanisms of alcohol consumption and CKD are not yet clear. Recently, McCarthy et al. ${ }^{40}$ reported that ethanol at low concentrations $(0.02-0.1 \mathrm{mmol} / \mathrm{ml})$ protected glomerular podocytes through alcohol dehydrogenase and 20-hydroxyeicosatetraenoic acid. Ethanol at high concentrations $(0.4 \mathrm{mmol} / \mathrm{ml})$ altered the actin cytoskeleton, induced CYP2e1, increased superoxide production and inhibited alcohol dehydrogenase gene expression. Ethanol at low concentrations upregulated the expression of alcohol dehydrogenase and CYP4a12a. In addition, 20-hydroxyeicosatetraenoic acid, an arachidonic acid metabolite generated by CYP4a12a, blocked the ethanol-induced cytoskeletal derangement and superoxide generation. Changes in podocyte 
Table 6 The associations of alcohol consumption and eGFR $<60 \mathrm{ml} / \mathrm{min} / 1.73 \mathrm{~m}^{2}$ in women stratified by smoking

\begin{tabular}{|c|c|c|c|c|c|c|c|c|}
\hline & \multicolumn{4}{|c|}{ Nonsmokers } & \multicolumn{4}{|c|}{ Smokers } \\
\hline & \multicolumn{2}{|c|}{ Univariate model } & \multicolumn{2}{|c|}{ Multivariate mode ${ }^{\beta}$} & \multicolumn{2}{|c|}{ Univariate model } & \multicolumn{2}{|c|}{ Multivariate mode ${ }^{\circledR}$} \\
\hline & OR $(95 \% \mathrm{Cl})$ & P-value & OR $(95 \% \mathrm{Cl})$ & P-value & OR $(95 \%$ CI) & P-value & OR $(95 \% \mathrm{Cl})$ & $P$-value \\
\hline Drinking rarely $(n(\%))$ & Reference & & Reference & & Reference & & Reference & \\
\hline occasionally & $0.84(0.74-0.96)$ & 0.011 & $0.74(0.64-0.86)$ & $<0.001$ & $0.71(0.39-1.29)$ & 0.258 & $0.61(0.29-1.30)$ & 0.195 \\
\hline$\leqq 19$ g/day & $0.92(0.89-0.95)$ & $<0.001$ & $0.94(0.91-0.97)$ & 0.001 & $0.93(0.80-1.09)$ & 0.363 & $0.90(0.75-1.07)$ & 0.24 \\
\hline 20-39 g/day & $0.65(0.59-0.70)$ & $<0.001$ & $0.66(0.60-0.72)$ & $<0.001$ & $0.61(0.49-0.76)$ & $<0.001$ & $0.55(0.42-0.72)$ & $<0.001$ \\
\hline 40-59 g/day & $0.58(0.49-0.70)$ & $<0.001$ & $0.53(0.43-0.65)$ & $<0.001$ & $0.52(0.37-0.72)$ & $<0.001$ & $0.51(0.34-0.75)$ & 0.001 \\
\hline$\geqq 60$ g/day & $0.42(0.29-0.62)$ & $<0.001$ & $0.35(0.23-0.54)$ & $<0.001$ & $0.29(0.16-0.50)$ & $<0.001$ & $0.27(0.14-0.54)$ & $<0.001$ \\
\hline Age $(\times 10$ years $)$ & $1.69(1.65-1.73)$ & $<0.001$ & $1.59(1.54-1.63)$ & $<0.001$ & $2.08(1.92-2.26)$ & $<0.001$ & $1.90(1.69-2.07)$ & $<0.001$ \\
\hline $\mathrm{BMI}\left(\times 10 \mathrm{~kg} / \mathrm{m}^{2}\right)$ & $1.51(1.45-1.58)$ & $<0.001$ & $0.77(0.73-0.81)$ & $<0.001$ & $1.40(1.17-1.67)$ & $<0.001$ & $0.76(0.59-0.97)$ & 0.026 \\
\hline Systolic BP (mm Hg) & $1.01(1.00-1.01)$ & $<0.001$ & $0.99(0.99-1.00)$ & $<0.001$ & $1.01(1.00-1.01)$ & $<0.001$ & $0.99(0.88-1.00)$ & 0.045 \\
\hline Diastolic BP (mm Hg) & $1.01(1.00-1.01)$ & $<0.001$ & $1.00(1.00-1.01)$ & 0.002 & $1.01(1.00-1.01)$ & 0.01 & $1.00(1.00-1.01)$ & 0.605 \\
\hline Triglyceride ( $\left.\log _{10} \mathrm{mg} / \mathrm{dl}\right)$ & $2.65(2.47-2.85)$ & $<0.001$ & $1.22(1.11-1.34)$ & $<0.001$ & $3.15(2.38-4.18)$ & $<0.001$ & $1.72(1.14-2.57)$ & 0.009 \\
\hline HDL-C $(\times 10 \mathrm{mg} / \mathrm{dl})$ & $0.92(0.91-0.93)$ & $<0.001$ & $1.01(0.99-1.02)$ & 0.239 & $0.88(0.85-0.92)$ & $<0.001$ & $0.99(0.93-1.04)$ & 0.606 \\
\hline Hemoglobin A1c (\%) & $1.05(1.02-1.07)$ & $<0.001$ & $0.86(0.83-0.89)$ & $<0.001$ & $1.17(1.08-1.26)$ & $<0.001$ & $0.97(0.86-1.09)$ & 0.673 \\
\hline Uric acid (mg/dl) & $1.99(1.96-2.02)$ & $<0.001$ & 2.02 (1.99-2.06) & $<0.001$ & $1.73(1.63-1.84)$ & $<0.001$ & $1.73(1.62-1.85)$ & $<0.001$ \\
\hline \multicolumn{9}{|l|}{ Past history of } \\
\hline Heart disease & $1.57(1.48-1.67)$ & $<0.001$ & $1.23(1.15-1.32)$ & $<0.001$ & $2.43(1.88-3.13)$ & $<0.001$ & $1.50(1.11-2.03)$ & 0.009 \\
\hline Stroke & $1.70(1.58-1.84)$ & $<0.001$ & $1.36(1.25-1.49)$ & $<0.001$ & $1.82(1.25-2.65)$ & 0.002 & $0.98(0.62-1.52)$ & 0.913 \\
\hline
\end{tabular}

Abbreviations: BMI, body mass index; BP, blood pressure; Cl, confidence interval; eGFR, estimated glomerular filtration rate; HDL-C, high-density lipoprotein cholesterol; OR, odds ratio.

${ }^{a}$ Adjusted for alcohol consumption, age, body mass index, mean arterial pressure, triglyceride level, HDL cholesterol level, hemoglobin A1c, uric acid, past history of heart disease and stroke.

structure and the resulting alterations in glomerular filtration barrier function may be early events in the progressive loss of glomerular function and the onset of proteinuria and chronic disease. Ethanol may positively and negatively affect several metabolites and signaling pathways involved in regulating glomerular barrier function. ${ }^{40}$

There are several limitations to this study. First, we could not infer a causal relationship between drink scores and proteinuria and eGFR because of the cross-sectional nature of the study. Further studies are needed to examine the causality of our findings. Second, the presence of proteinuria was determined by a single measurement using a dipstick test that may have led to misclassifications. Prior studies have shown that even a single dipstick indication of proteinuria is a significant risk factor for all-cause mortality, cardiovascular mortality ${ }^{41,42}$ and ESRD. ${ }^{43,44}$ Third, the drinking and smoking habits of the participants were evaluated only by a self-reported questionnaire. Therefore, a reporting bias may have existed in the entire cohort. However, because the participants did not benefit from answering the questions inaccurately or untruthfully, accurate information was expected. Fourth, we did not have information on the type of alcoholic beverage. Some characteristics have been reported to be because of the type. For example, long-term exposure to polyphenolrich red wine has been shown to lead to an enhancement of antioxidant defenses in rat plasma and kidney. ${ }^{45}$ Fifth, there is a huge potential for selection bias in this study, with nearly $60 \%$ excluded, mostly because of missing data on the effect modifier and exposure. Sixth, only current smoking was assessed; this habit may change as individuals become ill and hence past smoking may be more relevant to current CKD risk.

In this cross-sectional study, mild to moderate alcohol consumption was associated with a lower prevalence of proteinuria, but this association was slightly attenuated in smokers. Furthermore, alcohol consumption was inversely associated with the risk of a decreased eGFR. We suspect that mild to moderate alcohol consumption may be associated with a lower risk of CKD (proteinuria and eGFR), especially among nonsmokers, because smoking might have modified the potential benefits of alcohol to prevent CKD.

\section{CONFLICT OF INTEREST}

The authors declare no conflict of interest.

\section{ACKNOWLEDGEMENTS}

This work was supported by the Health and Labor Sciences Research, Grants for 'Research on the positioning of CKD in Specific Health Check and Guidance in Japan' (20230601), the Ministry of Health, Labor and Welfare of Japan.

1 Go AS, Chertow GM, Fan D, McCulloch CE, Hsu CY. Chronic kidney disease and the risks of death, cardiovascular events, and hospitalization. N Engl J Med 2004; 351: 1296-1305.

2 Keith DS, Nichols GA, Gullion CM, Brown JB, Smith DH. Longitudinal follow-up and outcomes among a population with chronic kidney disease in a large managed care organization. Arch Intern Med 2004; 164: 659-663.

3 Obi Y, Kimura T, Nagasawa Y, Yamamoto R, Yasuda K, Sasaki K, Kitamura H, Imai E, Rakugi $\mathrm{H}$, Isaka $\mathrm{Y}$, Hayashi T. Impact of age and overt proteinuria on outcomes of stage 3 to 5 chronic kidney disease in a referred cohort. Clin J Am Soc Nephrol 2010; 5: 1558-1565.

4 National Kidney Foundation. K/DOQI clinical practice guidelines for chronic kidney disease: evaluation, classification, and stratification. Am J Kidney Dis 2002; 39 (2 Suppl 1): S1-266.

5 Nagasawa Y, Yamamoto R, Rakugi H, Isaka Y. Cigarette smoking and chronic kidney diseases. Hypertens Res 2012; 35: 261-265.

6 Yamamoto R, Nagasawa Y, Shoji T, Iwatani H, Hamano T, Kawada N, Inoue K, Uehata T, Kaneko T, Okada N, Moriyama T, Horio M, Yamauchi A, Tsubakihara Y, Imai E, Rakugi H, Isaka Y. Cigarette smoking and progression of IgA nephropathy. Am J Kidney Dis 2010; 56: 313-324.

7 Schaeffner E, Ritz E. Alcohol and kidney damage: a Janus-faced relationship. Kidney Int 2012; 81: 816-818.

8 Yamamoto R, Nagasawa Y, Iwatani H, Shinzawa M, Obi Y, Teranishi J, Ishigami T, Yamauchi-Takihara K, Nishida M, Rakugi H, Isaka Y, Moriyama T. Self-reported sleep 
duration and prediction of proteinuria: a retrospective cohort study. Am J Kidney Dis 2012; 59: 343-355.

9 Robinson-Cohen C, Katz R, Mozaffarian D, Dalrymple LS, de Boer I, Sarnak M, Shlipak M, Siscovick D, Kestenbaum B. Physical activity and rapid decline in kidney function among older adults. Arch Intern Med 2009; 169: 2116-2123.

10 Wakasugi M, Kazama JJ, Yamamoto S, Kawamura K, Narita I. A combination of healthy lifestyle factors is associated with a decreased incidence of chronic kidney disease: a population-based cohort study. Hypertens Res 2013; 36: 328-333.

11 Bleyer AJ, Shemanski LR, Burke GL, Hansen KJ, Appel RG. Tobacco, hypertension, and vascular disease: risk factors for renal functional decline in an older population. Kidney Int 2000; 57: 2072-2079.

12 Brancati FL, Whelton PK, Randall BL, Neaton JD, Stamler J, Klag MJ. Risk of end-stage renal disease in diabetes mellitus: a prospective cohort study of men screened for MRFIT. Multiple Risk Factor Intervention Trial. JAMA 1997; 278 : 2069-2074.

13 Carter BD, Abnet CC, Feskanich D, Freedman ND, Hartge P, Lewis CE, Ockene JK, Prentice RL, Speizer FE, Thun MJ, Jacobs EJ. Smoking and mortality-beyond established causes. N Engl J Med 2015; 372: 631-640.

14 Tozawa M, Iseki K, Iseki C, Oshiro S, Ikemiya Y, Takishita S. Influence of smoking and obesity on the development of proteinuria. Kidney Int 2002; 62: 956-962.

15 Nicholson JP, Teichman SL, Alderman MH, Sos TA, Pickering TG, Laragh JH. Cigarette smoking and renovascular hypertension. Lancet 1983; 2: 765-766.

16 Ritz E, Benck U, Franek E, Keller C, Seyfarth M, Clorius J. Effects of smoking on renal hemodynamics in healthy volunteers and in patients with glomerular disease. J Am Soc Nephrol 1998; 9: 1798-1804.

17 Blann AD, McCollum CN. Adverse influence of cigarette smoking on the endothelium. Thromb Haemost 1993; 70: 707-711.

18 Briasoulis A, Agarwal V, Messerli FH. Alcohol consumption and the risk of hypertension in men and women: a systematic review and meta-analysis. J Clin Hypertens (Greenwich) 2012; 14: 792-798.

19 Baliunas DO, Taylor BJ, Irving H, Roerecke M, Patra J, Mohapatra S, Rehm J. Alcohol as a risk factor for type 2 diabetes: A systematic review and meta-analysis. Diabetes Care 2009; 32: 2123-2132.

20 Roerecke M, Rehm J. The cardioprotective association of average alcohol consumption and ischaemic heart disease: a systematic review and meta-analysis. Addiction 2012; 107: $1246-1260$.

21 Ronksley PE, Brien SE, Turner BJ, Mukamal KJ, Ghali WA. Association of alcohol consumption with selected cardiovascular disease outcomes: a systematic review and meta-analysis. BMJ 2011; 342: d671.

22 Gunzerath L, Faden V, Zakhari S, Warren K. National Institute on Alcohol Abuse and Alcoholism report on moderate drinking. Alcohol Clin Exp Res 2004; 28 829-847.

23 Nagasawa Y, Yamamoto R, Shinzawa M, Hasuike Y, Kuragano T, Isaka Y, Nakanishi T, Iseki K, Yamagata K, Tsuruya K, Yoshida H, Fujimoto S, Asahi K, Moriyama T, Watanabe $T$. Body mass index modifies an association between self-reported regular exercise and proteinuria. J Atheroscler Thromb 2015; 23: 402-412.

24 Menon V, Katz R, Mukamal K, Kestenbaum B, de Boer IH, Siscovick DS, Sarnak MJ, Shlipak MG. Alcohol consumption and kidney function decline in the elderly: alcohol and kidney disease. Nephrol Dial Transplant 2010; 25: 3301-3307.

25 Reynolds K, Gu D, Chen J, Tang X, Yau CL, Yu L, Chen CS, Wu X, Hamm LL, He J. Alcohol consumption and the risk of end-stage renal disease among Chinese men. Kidney Int 2008; 73: 870-876.

26 White SL, Polkinghorne KR, Cass A, Shaw JE, Atkins RC, Chadban SJ. Alcohol consumption and 5-year onset of chronic kidney disease: the AusDiab study. Nephrol Dial Transplant 2009; 24: 2464-2472.

27 Yamagata K, Ishida K, Sairenchi T, Takahashi H, Ohba S, Shiigai T, Narita M, Koyama A. Risk factors for chronic kidney disease in a community-based population: a 10-year follow-up study. Kidney Int 2007; 71: 159-166.
28 Shankar A, Klein R, Klein BE. The association among smoking, heavy drinking, and chronic kidney disease. Am J Epidemiol 2006; 164: 263-271.

29 Matsuo S, Imai E, Horio M, Yasuda Y, Tomita K, Nitta K, Yamagata K, Tomino Y, Yokoyama $\mathrm{H}$, Hishida A. Revised equations for estimated GFR from serum creatinine in Japan. Am J Kidney Dis 2009; 53: 982-992.

30 Arima H, Tanizaki Y, Kiyohara Y, Tsuchihashi T, Kato I, Kubo M, Tanaka K, Ohkubo K, Nakamura H, Abe I, Fujishima M, lida M. Validity of the JNC VI recommendations for the management of hypertension in a general population of Japanese elderly: the Hisayama study. Arch Intern Med 2003; 163: 361-366.

31 Seino $\mathrm{Y}$, Nanjo K, Tajima N, Kadowaki T, Kashiwagi A, Araki E, Ito C, Inagaki N, Iwamoto Y, Kasuga M, Hanafusa T, Haneda M, Ueki K. Report of the committee on the classification and diagnostic criteria of diabetes mellitus. J Diabetes Investig 2010; 1: 212-228.

32 Hata Y, Mabuchi H, Saito Y, Itakura H, Egusa G, Ito H, Teramoto T, Tsushima M, Tada N, Oikawa S, Yamada N, Yamashita S, Sakuma N, Sasaki J. Report of the Japan Atherosclerosis Society (JAS) Guideline for Diagnosis and Treatment of Hyperlipidemia in Japanese adults. J Atheroscler Thromb 2002; 9: 1-27.

33 Perneger TV, Whelton PK, Puddey IB, Klag MJ. Risk of end-stage renal disease associated with alcohol consumption. Am J Epidemiol 1999; 150: 1275-1281.

34 Klein R, Klein BE, Tomany SC, Moss SE. Ten-year incidence of age-related maculopathy and smoking and drinking: the Beaver Dam Eye Study. Am J Epidemiol 2002; 156: 589-598.

$35 \mathrm{Hsu}$ YH, Pai HC, Chang YM, Liu WH, Hsu CC. Alcohol consumption is inversely associated with stage 3 chronic kidney disease in middle-aged Taiwanese men. BMC Nephrol 2013; 14: 254.

36 Sato KK, Hayashi T, Uehara S, Kinuhata S, Oue K, Endo G, Kambe H, Fukuda K. Drinking pattern and risk of chronic kidney disease: the Kansai Healthcare Study. Am J Nephrol 2014; 40: 516-522.

37 Schaeffner ES, Kurth T, de Jong PE, Glynn RJ, Buring JE, Gaziano JM. Alcohol consumption and the risk of renal dysfunction in apparently healthy men. Arch Intern Med 2005; 165: 1048-1053.

38 Gaziano JM, Buring JE, Breslow JL, Goldhaber SZ, Rosner B, VanDenburgh M, Willett W, Hennekens $\mathrm{CH}$. Moderate alcohol intake, increased levels of high-density lipoprotein and its subfractions, and decreased risk of myocardial infarction. N Engl J Med 1993; 329: 1829-1834.

39 Ridker PM, Vaughan DE, Stampfer MJ, Glynn RJ, Hennekens CH. Association of moderate alcohol consumption and plasma concentration of endogenous tissue-type plasminogen activator. JAMA 1994; 272: 929-933.

40 McCarthy ET, Zhou J, Eckert R, Genochio D, Sharma R, Oni O, De A, Srivastava T, Sharma R, Savin VJ, Sharma M. Ethanol at low concentrations protects glomerular podocytes through alcohol dehydrogenase and 20-HETE. Prostaglandins Other Lipid Mediat 2015; 116-117: 88-98.

41 Brantsma AH, Bakker SJ, de Zeeuw D, de Jong PE, Gansevoort RT. Extended prognostic value of urinary albumin excretion for cardiovascular events. J Am Soc Nephrol 2008; 19: 1785-1791.

42 Zambon S, Maggi S, Zanoni S, Romanato G, Noale M, Corti MC, Sartori L, Musacchio E, Baggio G, Sergi G, Crepaldi G, Manzato E. Association of single measurement of estimated glomerular filtration rate and non-quantitative dipstick proteinuria with all-cause and cardiovascular mortality in the elderly. Results from the Progetto Veneto Anziani (Pro.V.A.) Study. Atherosclerosis 2012; 220: 201-207.

43 Iseki K, Ikemiya Y, Iseki C, Takishita S. Proteinuria and the risk of developing end-stage renal disease. Kidney Int 2003; 63: 1468-1474.

44 Ishani A, Grandits GA, Grimm RH, Svendsen KH, Collins AJ, Prineas RJ, Neaton JD. Association of single measurements of dipstick proteinuria, estimated glomerular filtration rate, and hematocrit with 25-year incidence of end-stage renal disease in the multiple risk factor intervention trial. J Am Soc Nephrol 2006; 17: 1444-1452.

45 Rodrigo R, Rivera G, Orellana M, Araya J, Bosco C. Rat kidney antioxidant response to long-term exposure to flavonol rich red wine. Life Sci 2002; 71: 2881-2895.

Supplementary Information accompanies the paper on Hypertension Research website (http://www.nature.com/hr) 